\title{
Derivational Grammar Model and Basket Verb: A Novel Approach to the Inflectional Phrase in the Generative Grammar and Cognitive Processing
}

\author{
Rajdeep Singh ${ }^{1}$ \\ ${ }^{1}$ Department of Linguistics, Payame Noor University, Tehran, Iran \\ Correspondence: Rajdeep Singh Department of Linguistics, Payame Noor University, Tehran, Iran.
}

Received: April 21, 2018

doi:10.5430/elr.v7n2p9
Accepted: June 6, 2018

Online Published: June 10, 2018

URL: https://doi.org/10.5430/elr.v7n2p9

\begin{abstract}
Generative grammar was a true revolution in the linguistics. However, to describe language behavior in its semantic essence and universal aspects, generative grammar needs to have a much richer semantic basis. In this paper, we took a novel morpho-syntactic approach to the inflectional phrase to account for the very diverse inflectional phrase qualities in different languages. Some languages show a very different surface verbal inflection, providing evidence of a different mental processing at the semantic level. In fact, the inflectional phrase is a great representative of the mental and semantic processing layers in mind. Therefore, in this study, we analyzed the inflectional phrase with a novel approach to take into account this rich verbal inflectional configuration in languages, and to describe why some languages behave in a different way in the spatial and temporal aspect. In this study, we analyzed and discussed the verbal inflectional structure of several languages, including German, Swahili, Persian, English, and Indonesian, and our result is the introduction of a semantic model which provides a much richer insight to the semantics/syntax interplay.
\end{abstract}

Keywords: generative grammar, verbal inflection, comparative study, Swahili, Persian, Spanish, semantics, syntax, cognitive processing

\section{Introduction to Derivational Grammar}

Generative grammar provides a great explanatory model to account for the variety and differences found amongst languages and even experts of generative grammar school investigated the semantics, providing a semantic component for syntactic structures (Jackendoff, 1975). Hanink (2018) investigates the post-syntactic inflection of the degree phrase, rather in the adjective level, providing a good model for the interplay between morphology and mind processing in the adjective phrase level, though Hanink does not go in depth in other aspects of Syntactic representation in mind. Bozic and Marslen-wilson (2010) investigate the distribution and the nature of grammatical computations across language processing networks by comparing complex inflectional words and minimal phrases with simple words. For the syntax, we should consider the morphology role and existing internal and external side for words is a good evidence of such a deep connection, in which, the external part relates to the fact that words play in group to form the meaning and final output, through syntax, and the internal side, relating to the phonological side of morphology, which complements the external side of syntax (Bleser, Bayer, \& Luzzatti, 1996). Generative grammar puts emphasis on the syntax, and it considers the morphology less important. However, in semantics, morphology plays a very strong role. Trueswell, Tanenhaus, \& Garnsey (1994) show how semantics influences on parsing. They use a different combination of ambiguous verbs and animate/inanimate effects on the syntactic interpretation.

The interplay between syntax and semantics has been an important field to invest as there is a big interest in semiotics. Hagoort (2003) investigates the effects of combined syntax and semantics to determine their influence on comprehension. He compared the combined effects of semantic and syntactic violations with the effects of the single semantic or syntactic violations on language-related events. Brooke, Tofiloski, and Taboada (2009) research the Semantic aspect of language in a cross-linguistic sentiment analysis, in which they take into consideration not only the semantics and syntax but also psycholinguistic factors.

Slobin (1966) compares abstract semantic concepts to see whether there is a specific mental world where each speaker lives in and influenced by it. However, there is a lack of a much more nuanced approach to syntax, with 
semantics at its core. Chafe (1970) investigates the meaning, structure, grammaticality and he works through the criteria of sentences which we judge well-formed at the semantic level. Despite the difficulty measuring the mental structure and processing in the final output form of utterances, De Villiers, \& Pyers (2002) investigates the false belief and its develping phases with various measures of spontaneous language production and comprehension in a longitudinal study, and they find a good correlation between syntax and false-belief-understanding. Bloom (1994) works on the role of syntax-semantic mappings in nominal group acquisition and overall lexical development. Ferreira (1999) proposes a tree adjoining model, in which one can access to all possible elementary trees for a verb lemma to figure out the only remained activated clausal elementary tree, which will determine the ultimate form of the sentence. Despite wide agreement on the constraints that one may use during the interpretation and formulation of sentences, how one implements these constraints in the overall semantic/syntactic machinery is a matter of debate in psycholinguistics. Bierwisch (1967) analyzes the syntactic features in morphology and proposes a model in which one introduces inflections by a set of morphological rules, intervening between the syntactic and the phonological component of the grammar. Though some have the view that sentence comprehension is autonomous and not influenced by the syntactic framework, Jackendoff (1975) shows the imposing syntax in all aspects of semantics and interpretation. In another study, Munnich, Landau, \& Dosher (2001) investigate another aspect of semantics, such as the spatial preposition, and its role in the semantics and syntax. Kratzer, \& Heim (1999) work through a model to investigate the role of semantics in the generative grammar since the notion of semantics and the explicit presence of inflection show great irregularities among languages. Slade and Ruffman (2005) investigate how language relates to the theory of mind. Slade and Ruffman (2011) provide a good predictive tool for working memory and false belief by coupling the longitudinal study of syntax with the analysis of semantics.

Bouchard (1995) investigates the semantics of the syntax itself, where he takes a minimalist approach to the grammar to show the symbolic representation of the meaning in the syntax (Bouchard, 1995). Singh (2018) investigates the specific spatial prepositions in the syntax with the type-logical method and relates the syntactic properties with the semantics of the surface structure.

In this study, we discuss the mutual influence of semantics on syntactic form and, the influence of the syntactic form on the semantics. In this approach, the verbal component plays the role of the linker between word classes, showing itself on the surface as an inflectional phrase which one encounters in some languages.

\subsection{Derivational Grammar Model Properties}

Based on Noam Chomsky's generative grammar parameters and principles, there is a finite set of common essential principles we can find are in all languages and also a finite set of parameters that determine syntactic variations amongst languages. Further, in principles and parameters, the inflectional phrase is an important and essential element which is a functional phrase that has inflectional properties (Chomsky, 2005).

In generative grammar, there are two main processing structures, i.e. deep structure and surface structure, which underlie sentences in any language. In some languages, one inflects verbs for tense and subject agreement and based on generative grammar, all these infections exist under one category, inflectional phrase. What this study purports to clarify is this very inflectional phrase. We propose a model which provides another explanation for the present inflectional properties in the verb. The inflectional properties provide the semantic and lexical properties of the words. The Derivational grammar (DG) model expresses that there are languages with different levels of inflectional properties and these inflectional properties do not comprise only subject agreement and tense, but also object agreement, adverbial agreement, and even spatial agreement. In DG model, we divide the deep structure further into two layers semantic-lexical layer and derivational level. In this way, derivational grammar considers the connection between some word classes, such as between nouns and verbs, emphasizing that there should exist a strong connection between verbs and other word classes, and DG model a central role for the verb as a semantic linker. We call this central verb Basket verb (BV). Therefore, in the semantic layer, BV comprises the semantic qualities of other word classes.

Word classes which have a strong connection with the verb transfer their qualities to BV in the semantic layer. Therefore, we have the semiotics and semantics of the syntax in the semantic layer in mind, and the analysis of syntax should have a much broader semantic component. Because of the semantic processing of syntax in mind, in some languages the verbs appear with strong inflectional properties, presenting the evidence of a semantic verb. In this study, we chose languages which show very different inflectional phrase qualities for the analysis of their verb component and by choosing these languages; we present a more balanced and thorough perspective to the inflectional phrase. 
The generative grammar treats the mind processing. However, as we mentioned earlier, we should enrich the generative grammar further with the semantics, so we consider the internal structure of languages and the processing involved, the ones in the deep structure. For example, in the Indonesian language, no inflection presents in the main verb in contrast with some other inflected languages. To explain it further, we provide examples of Spanish and Indonesian, which are different in their verbal inflectional phrase properties. Example1 shows the structural differences between the non-verbal inflected language like Indonesian, and the verbal inflected one like Spanish.

1) Saya pergi ke toko kemarin.

I went to the shop yesterday.

Saya pergi ke toko besok.

I will go to the shop tomorrow.

Yo comprare algo hoy para mi familia.

I will buy something for my family today.

Yo compré algo para mi familia ayer.

I bought something for my family yesterday.

The temporal adverb has a significant contrastive role to show the tense in non-verbal inflected languages, like Indonesian, and therefore, on understands the tense by the existing temporal adverb. Therefore, we should consider the temporal adverb in the surface as a representative of BV of the deep structure, though adverb is not anymore part of the verb in the surface. Likewise, the subject and object agreement are the representatives of subject and object of the verb. Therefore, the subject/object part of BV detaches from the basket verb in the surface form.

In contrast, in Spanish, a highly inflected language, the verbal inflection shows the tense, having the temporal adverb as a clarifying element. It is noteworthy to point out that the subject agreement still exists in the surface verb, just as in $\mathrm{BV}$, but the object agreement component of the basket verb is no more present in the surface verb. The spatial adverb appears in some Spanish verbs like preveer, to forecast, and it is an evidence of a strong connection between verb and adverb in mind. The temporal aspect in Spanish appears in the surface verb, providing the evidence of a strong connection between the verb and temporal adverb in mind during forming BV. After describing facts about Spanish and Indonesian inflectional phrase, let's get back to the generative grammar and discuss why generative grammar does not account for the inflectional phrase. In generative grammar, one looks at the temporal as just a common adverb and there is no clear functional role for the temporal adverb inside the inflectional phrase. This is in sharp contrast with the fact that temporal adverb has part of the lost information about BV. The semantics of the temporal adverb shows the tense of the sentence while in generative grammar one treats adverbs as the adjunct and considers them as trivial in the sentence formation. To assume that verbal inflection happens based on the idiosyncratic form is far from a universal concept which generative grammar claims. As we will show, the temporal adverb and spatial adverb contribute to the semantics and to the syntactic form of the surface verb.

We can find another good example in German where many verbs have a spatial preposition in their verb root and these verbs have their spatial prepositional component detached from them in the surface verb. This is again another interesting fact that explains how the spatial preposition had a strong connection to the verb in the mental BV, but somehow loses its strong connection with the verb in the surface structure, detaching from the surface verb. In this study, we consider BV presence to be a universal principle and we show this by the morpho-syntactic approach. However, in some languages, this universal BV has its components detached from the surface verb, and in some other languages, the qualities related to different word classes are part of the verb semantics in the surface structure.

In this study, we propose a supplementary view to generative grammar which brings a clear descriptive and analytic insight to the grammar, providing a much richer explanation of the inflectional phrase in the generative grammar framework and the interplay between the syntax and semantics. We establish the semantic and syntactic relationship to explain the mind processing of the verbal component for different languages.

We analyze different languages to provide the evidence for our model and we combine the syntax and semantics so we analyze the linguistic processing in mind. In the following sections, we propose the concept of the Basket Verb as a linker in the deep structure.

\subsection{Analysis based on Derivational Grammar}

The morpho-syntactic approach is the basis of Derivational Grammar and DG explains the inflectional phrase in generative grammar and the mind processing before our utterances. The morpho-syntactic approach considers 
several layers in the syntax and semantics processing. Let's consider Figure 1 so we get a better idea of the whole process. In this model, different layers present different temporal processing sequences which influence and generate thought and utterances. Derivational Grammar model, DG, comprises three essential layers semantic-lexical, derivational and surface layer. Since derivational grammar is a generative grammar supplement, the first two layers Semantic-lexical and Derivational, are equivalent to the Deep structure in generative grammar, and the last layer surface layer, is equivalent to the surface structure in the generative grammar. Therefore, we analyze the surface layer further to phonological and logical form, just as Chomsky (2003) proposes this in generative grammar.

In figure 1, lexical categories or word classes are present with their own specific semantics in the semantic-lexical layer as information processing in mind begins, and this involves the access to word classes in the mental lexicon, and activation of all the categories like the noun, preposition, adjective, adverb, conjunction, determiner, pronoun, verb, and exclamation.

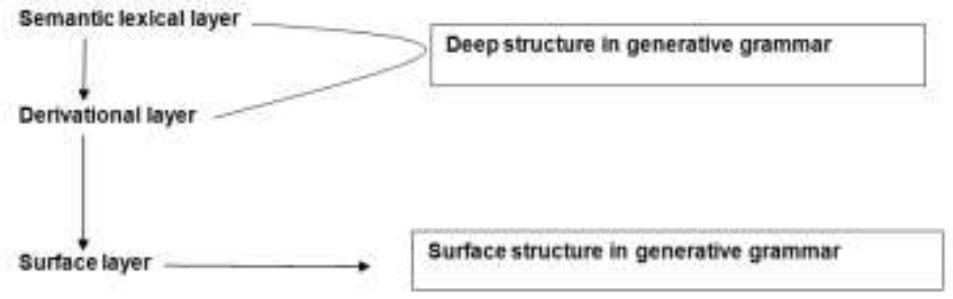

Figure 1. Language processing based on derivational model

In semantic-lexical layer, there is a stronger connection between the verb and other word classes like the noun, pronoun, the temporal adverb and a preposition. The connection between the verb and other word classes is of importance and we call this connection SC, which stands for Strong Connection. SC influences the verb in a way that qualities of other word classes, such as temporal, impose themselves in the verb in the derivational layer.

\section{Basket Verb}

The mind forms different connections between word classes in the derivational layer. However, the connection between the verb and other word classes is the dominant one: the verb shares qualities of other word classes in this layer. SC (Strong Connection) is much stronger than connections between other word classes, such as the one between the noun and the adverb. Therefore, SC will influence the form of the surface verb. The output of SC will be Basket verb, an abstract verb in mind. Basket Verb, BV, is an abstract and mental verb which comprises residues of the SC. Figure 2 shows how we consider the new arrangement of word classes.

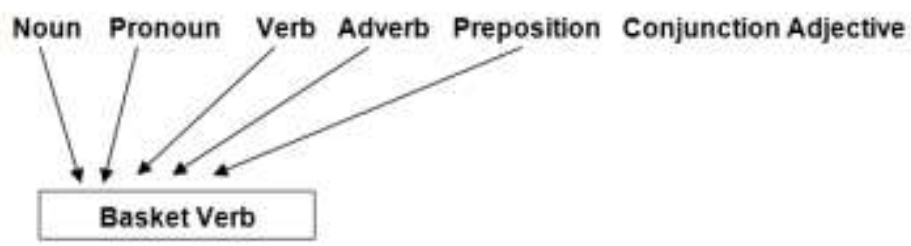

Figure 2. Basket verb representation

SC inserts and shares the characteristics of the noun, pronoun, the temporal adverb and preposition in to the verb, and this abstract mental BV is a representative and derivation of all the other categories. BV is still a functional verb though the semantic component of the other categories is strong in this mental verb. Noun and pronoun part of SC provides the future agreement of verbal inflections for subject and object in the utterance. Preposition and temporal adverb carry the future inflections for the verb, in spatial aspect and temporal tense. BV is a universal of languages, as no matter which language we look at, the BV formation is in the mental derivational layer, making the verb a representative of the whole sentence in the speaker's mind. However, this abstract of universal BV shows itself in 
surface structure in a different way. Therefore, it is important to consider the adverb and many other word classes as part of that mental BV, which may be separate from BV in the surface form. We show the relationship between BV and the surface verb in table 1 .

Table 1. Basket verb and surface verb qualities and processing

\begin{tabular}{lll} 
Verb type & Processing level & Relation to SC \\
Basket verb & Mental derivational level & Enriched with other word \\
Surface verb & Surface level & Lost qualities of SC \\
\hline
\end{tabular}

Let's consider the sentence 2 to show better the concept of Basked Verb.

1) They previewed the upcoming football season.

In this sentence, the verb "previewed" is an example of the utterance-ready reduced form of BV based on the phonological and the logical forms of the surface layer. Figure 3 shows the analysis of the verb "previewed" with DG model.
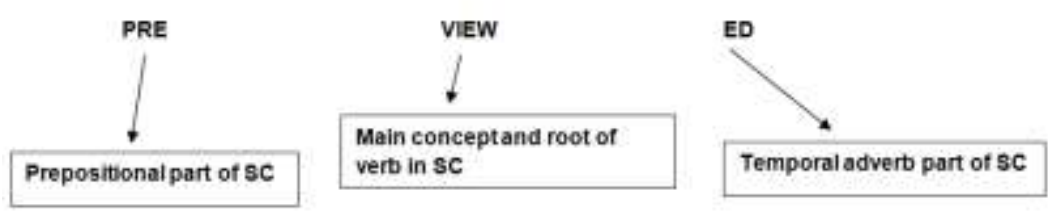

Figure 3. Analysis of the verb "previewed" through derivational grammar

As Figure 3 shows, the prepositional aspect has the potential to appear in the verbal inflections, though it happens in languages in an unpredictable way. BV has a special role in the whole sentence formation since it connects the verb with other word classes and the SC presents the properties of other word classes in BV form.

After the derivational layer, information processing continues in the surface layer. Surface layer functions similar to the surface structure in Generative grammar. So in the first two layers of the derivational model, the cognitive system processes the information in a way that $\mathrm{BV}$ becomes enriched with the semantics of other word classes in its identity. The cognitive process ends after phonological and logical form superimposition on the surface. To explain the whole system of derivational grammar, an example of Swahili language in sentence 3 can provide us with further clarification.

2) Mimi nimemwona mgeni wa kaka yangu leo.

I have seen my brother's guest today.

In Swahili language, verbal inflections comprise tense, subject and object agreement. In the analysis based on DG model, we assume that the final verb in the surface layer is a representative of BV in the Derivational layer, Deep structure. So the verb carries the identity of the temporal adverb and subject/object properties of noun/pronoun part of the sentence in the mental abstract layer. As we will show later, DG model analyzes the verbal part, where the latter is approximate of what BV would have been in mind. In Figure 4 we analyze the verb, nimemwona, in sentence 3 . 


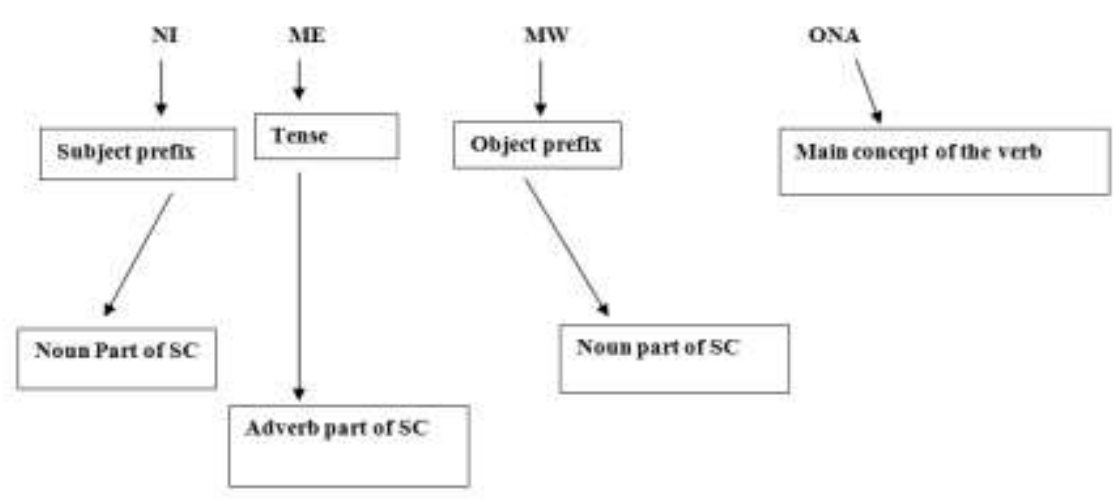

Figure 4. Derivational grammar based analysis of the verb component of sentence 3 (nimemwona)

The DG model can help clarify the issue in the languages which do not have a tense and subject/object agreement marked verbs. Here, the situation is reverse to what happens in Swahili, which is a verbal inflected language. To explain this point further, we shall consider sentence 4 from the Indonesian language.

1) Aku pergi ke toko ku kemarin.

I went to the shop yesterday.

In Figure 5, we analyze the verbal component of sentence 4 based on the derivation grammar approach.

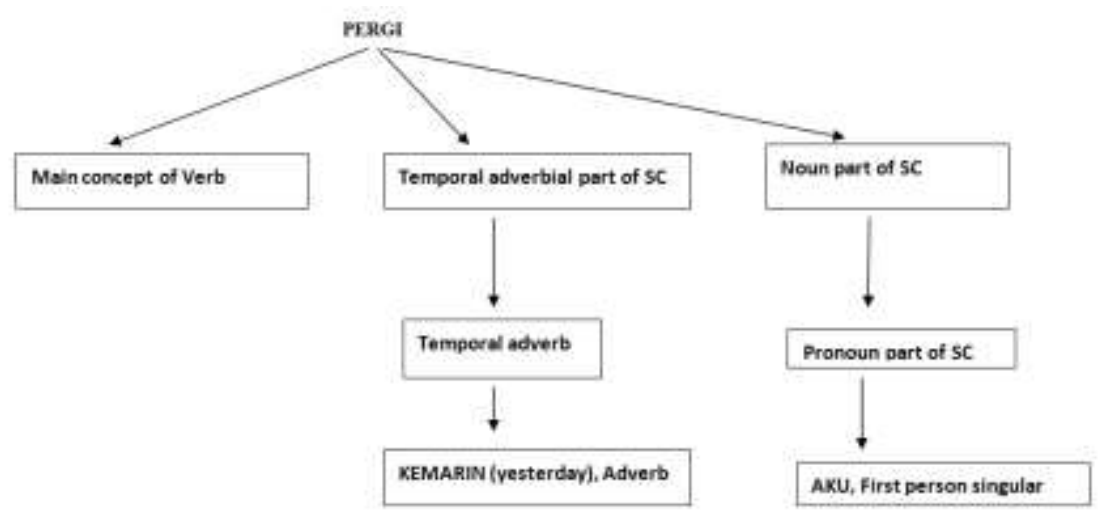

Figure 5. Derivational analysis of the verbal component of sentence 4 (pergi)

It is important to point out that in the analysis of a verbal component in the sentence 4, the temporal adverb part of SC (Strong connection) is not present in the verb, but temporal adverb, kemarin (yesterday), provides the temporal adverb aspect of SC in the derivational and semantic-lexical layers of DG model. Likewise, we show the subject agreement with the verb by the clear subject at the sentence initial, aku (first person pronoun), therefore no inflection happens in the surface structure. The final verb in the surface structure is the representative of BV in the deep structure, showing that in some languages, there is no need to overemphasize the temporal aspect where they exclude the temporal and subject/object agreement inflections from BV. An example from the English can be helpful to explain the role of BV better. Let's consider the sentence 5, and the verbal component analysis in Figure 6 based on the DG model. 
2) I showed him the way.
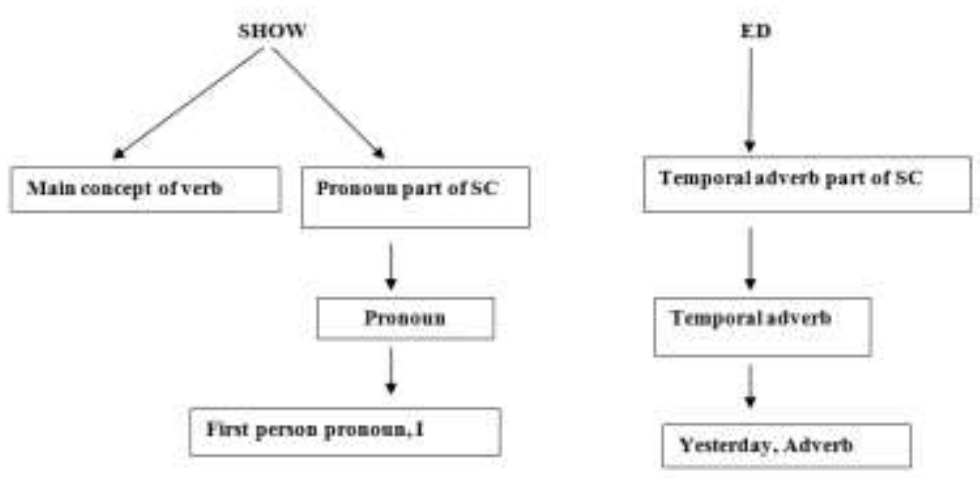

Figure 6. Derivational analysis of the verbal component of the sentence 5 (showed)

We can provide further evidence for prepositional (spatial) aspect of BV in languages such as German and Persian. In German and Persian, many verbs still carry their spatial aspect, and this spatial, prepositional aspect is the residue of the SC (Strong Connection) in the semantic-lexical layer. To understand better the spatial, prepositional aspect of BV, we consider example sentences 6 and 7 in German and Persian,.

6) Gegen ende des sommers, wird uns das geld ausgehen.

So by the summer, we will run out of money.

7) Man biroon raftam.

I went out

Figure 7 and Figure 8 show the verb analysis in sentence 6 and sentence 7.

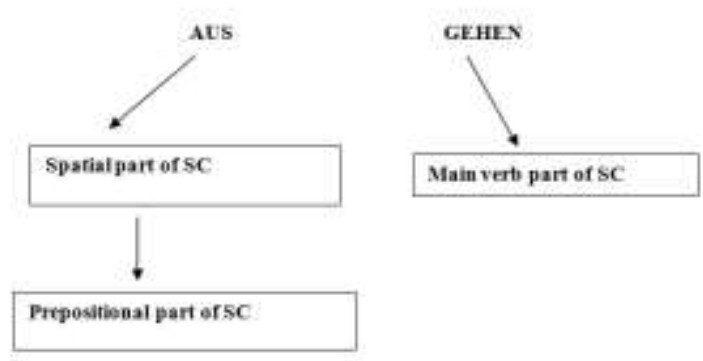

Figure 7. Derivational analysis of the verbal component (spatial aspect) in sentence 6 (ausgehen)
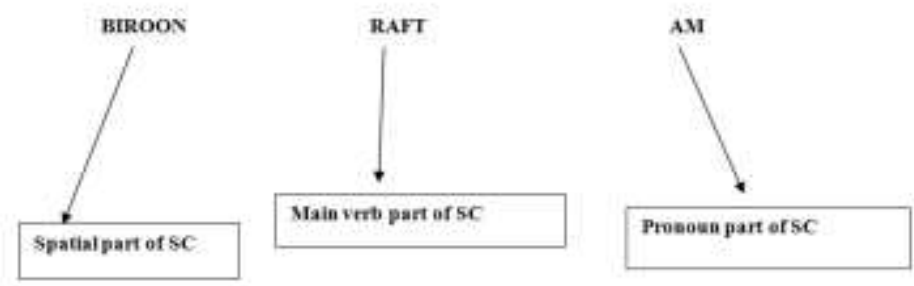

Figure 8. Derivational analysis of the verbal component (spatial aspect) of sentence 7 (biroonraftam)

In German aus, "out" in English, is a normal spatial preposition in German, while Biroun in Persian, is a common spatial preposition which means "out or out of". In German, the spatial verbal form has interesting features we will consider. As we showed in figure 7, the verb in German can have many spatial components as aus, in, zu and many 
others. In German, it has two prepositional components pointing to the same concept. Consider the example sentence 8 and the derivational grammar analysis of its verbal component geht aus in figure 9.

6) Er geht aus dem hause aus.

He goes out of the house.

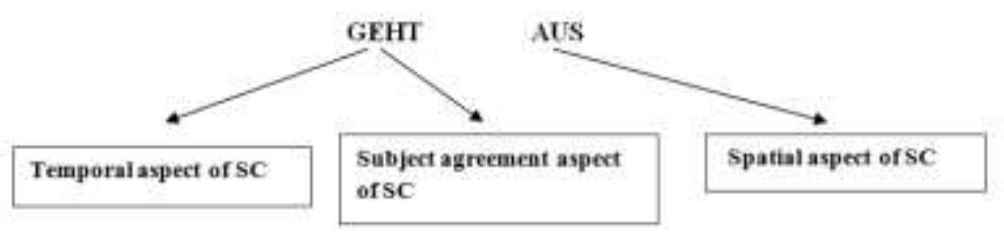

Figure 9. Derivational analysis of the verbal component ausgehen in sentence 8 (geht aus)

As we see in figure 9, the basic form of the verb in sentence 8 was ausgehen. However, in the surface structure, the spatial component detaches itself from the verbal form. The spatial component and the surface verbs are far from one another where the former is the semantic representation of the underlying BV in deep structure, and, two spatial adverb elements appear in the same sentence, both showing the same spatial quality. A further example of the Swahili may show another interesting evidence of such an abstract mental verb in the semantic/lexical layer in mind. Let's consider the example 9 and the figure 10 in the analysis of the verbal component.

6) Wewe utaendaje shuleni kesho?

How will you go to school tomorrow?
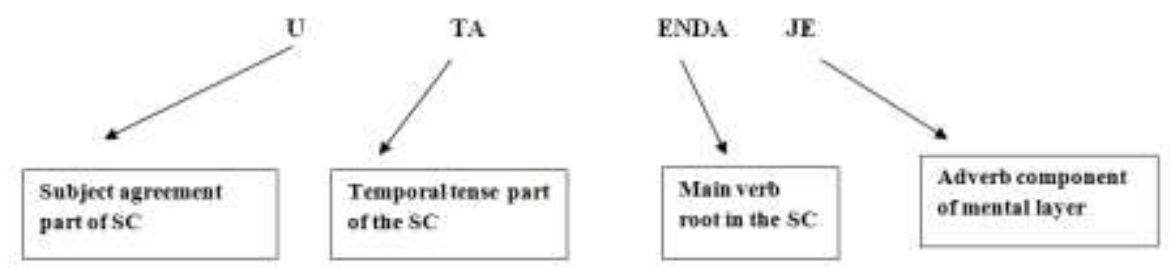

Figure 10. Analysis of verbal inflection of the verb utaendaje

As we mentioned, while analyzing the verbal component of the sentence 9, utaendaje, the adverb inflectional component is distinct from the temporal adverb. This adverb component, je, which means how, can become detached from the verb in other occasions, while changing the meaning to "whether". Consider example 10 and the figure 11 as the analysis of the verbal component in the sentence 10 .

6) Je utaenda shuleni kesho? Will you go to school tomorrow?
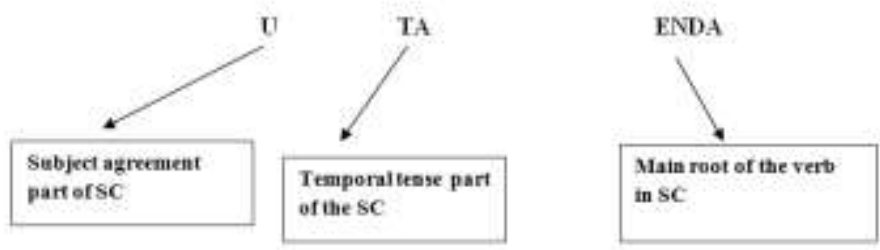

Figure 11. Analysis of the verbal component "utaenda" in sentence 10

Let's consider figure 11 and figure 10, the adverb component of BV can become detached from the verb in the surface layer in the mental layer, based on the semantics of the sentence. It is a good example of how some components of $\mathrm{BV}$, such as the adverb still appear in some languages, while these kinds of connections are not a common SC in the semantic/lexical layer. This reflects the rich quality of BV in its original form, in the mental layer. The same phenomenon happens in many other languages, though the semantic layer adverb is not part of the verb. Consider example 11 and notice the detached adverb component "aya" from the verb, used for the yes/no question formation. 


\section{8) Aya dirooz khoob khabidi?}

Did you sleep well yesterday?

So, as many examples pointed out the mental aspect of the verb, the Strong Connection and the resulted BV appear in the surface layer of different languages in a clear different way. In a language like Swahili, BV appears with some modifications, in the output of a sentence, having the temporal, subject /object agreement inflections in the final surface verb. While in English, the basket verb is semi-obscured, appearing with tense but limited subject agreement inflection. In a language like Indonesian, the surface verb is different compared to its mental basket verb and almost all the inflections are absent, though the broader syntax provides the semantic components necessary to utter meaningful and complete sentences, through the temporal adverb, noun, and pronoun. Therefore, in generative grammar analysis, it is vital to reconsider the inflectional phrase, bringing in the other possible inflectional identities in the inflectional phrase, such as the temporal adverb and spatial aspect. By doing this, we account for the universality of the grammar description and we form a much richer, all-encompassing view. The mind processing of $\mathrm{BV}$ will be an important clue for further research regarding the cognitive ability in processing languages and one can get a much better insight to this mental, abstract process through a thorough analysis of BV and the strong connection in the derivational and the semantic layer.

\section{Implications of Derivational Model}

We have a much richer context to interpret the sentence with through the DG model. BV undergoes changes in the surface form, being detached from its inflectional components, while once they were all connected to the BV inflectional phrase at the mental level. Therefore, we should regard the adverbial part as part of the BV inflectional phrase, where the former may become lost from the verb in the surface form. In fact, since the other components of the sentence were all once part of the BV inflectional phrase, we can build up the basic mental frame for the sentence. This analysis of the sentence is much closer to the way one processes information, by lowering the processing load, making it much more straightforward and easier. It gives us better insight into the way children learn language and language acquisition. By building the abstract BV, we get closer to that mental framework children make to express meaning, while playing with words in mind. To investigate language acquisition, we should move away from analyzing only the surface verbal component, and put emphasis on the analysis of the abstract BV. BV is a universal verb which encompasses other word classes, lowering the processing load, making language acquisition fast and easier. One application of the discovery of BV and the whole DG model is to use them to reconstruct what children make in their mind when they learn the language. In fact, instead of putting emphasis on the surface structure, we can concentrate on BV. Reconstructing BV is the key to language acquisition and mental processing. Only BV can absorb other word classes in the form of the inflectional phrase, giving cohesion and solidity to the meaning in mind.

\section{Conclusion and Result Discussion}

The derivational Grammar model is a processing model of the verbal component of the Generative grammar, explaining well how and why verbs in some languages behave in a different way. The verb does not have the inflection for the tense in many languages, being a big dilemma, how to account for this phenomenon in the generative grammar. In Derivational grammar, BV plays a very prominent role.

$\mathrm{BV}$ is a universal of language and it comes from SC in mind processing when we have words extracted from the mental lexicon and put in empty slots to provide the form for the semantics and meaning. If Basket Verb were to appear in the surface form of a language, in its fullest form, the tense and subject agreement, even object agreement, the spatial prepositional aspect and the adverb would have shown their identities through a series of inflections of the main verb in the general way.

Some languages like Swahili and German language, have their surface verb somewhat close to their BV form in the surface of their syntax, though still far from it. Some languages, like English and Spanish, have their BV semi-obscured in the surface form. In some others, the surface verb loses almost all the inflectional properties of the basket verb. However, we can build up and construct the BV by the present subject and temporal adverb. Therefore, the Basket verb is universal and present in all languages in the mental processing level. The surface verbal inflection in the output is a parameter and language specific, showing the temporal adverb, subject and object agreement in the surface form. Therefore, it is vital to consider the inflectional phrase of generative grammar as the representative of $\mathrm{BV}$. While providing this novel approach to the semantics and syntax, it is vital to research this abstract verb form further as it universally explains the thought process.

By introducing BV, there are ways to improve the semantics of inflectional phrase aspect of generative grammar. Through the derivational morpho-syntactic approach contribution, we can consider the temporal adverb, spatial adverb, the object, and many other word classes in the sentence as part of the inflectional phrase being detached from 
BV in the surface form. Generative grammar considers the subject/object agreement and does not deal with other connections that exist between word classes and BV. Derivational grammar deals with these connections and provides a rich picture of mind processing. The spatial prepositions and temporal adverbs provide the spatial aspect and temporal tense of the verb. Therefore, we can investigate the Spatial and temporal aspect in different languages. By doing this, we can account for the presence or absence of implicit temporal and spatial aspect in the inflected verbs which have been an important aspect in the analysis of languages and applied linguistics.

Basket BV is a very important feature in mind processing where neurons transfer signals, affecting the way people speak in different languages. It is possible to research BV formation through the surface structure of phrases and to find out the features that derive the surface verb from BV, the former being the actual verb in the mental level. As we discussed throughout this paper, the DG model provides an interesting insight into the way the mind processes information, and it explains how the semantics exerts pressure on the form of languages. By accounting for the adverbial aspect of the language, such as the spatial aspect, the DG model brings a rich supplement to the Generative grammar.

\section{Acknowledgements}

This work is done with great care and I am thankful to my family and other colleagues for the support that I needed for such a detailed and multi-lingual work.

\section{References}

Bierwisch, M. (1967). Syntactic features in morphology: General problems of so-called pronominal inflection in German. In to Honour Roman Jacobson, 239-270. The Hague: Mouton. https://doi.org/10.1515/9783111604763-022

Bleser, R., Bayer, J., \& Luzzatti C. (1996). Linguistic theory and morphosyntactic impairments in Italian and German aphasics. Journal of Neurolinguistics, 9, 3.-S.175-185. https://doi.org/10.1016/0911-6044(96)00007-3

Bloom, P. (1994). Possible names: The role of syntax-semantics mappings in the acquisition of nominals. Lingua, 92, 297-392, North Holland. https://doi.org/10.1016/0024-3841(94)90345-x

Bouchard, D. (1995). The semantics of syntax: A minimalist approach to grammar. University of Chicago Press. https://doi.org/10.1075/sl.23.3.18san

Bozic, M., \& Marslen-Wilson, W. D. (2010). Neurocognitive Contexts for Morphological Complexity: Dissociating Inflection and Derivation. Language and Linguistics Compass, 4(11), 1063-1073. https://doi.org/10.1111/j.1749-818x.2010.00254.x

Brooke, J., Tofiloski, M., \& Taboada, M. (2009). Cross-linguistic sentiment analysis: from English to Spanish. International conference RANLP. Retrieved from: http://www.aclweb.org/anthology/R09-1010

Chafe, W. L. (1970). Meaning and the structure of language. The University of Chicago Press, Illinois, 60637. https://doi.org/10.1353/sls.1972.0003

Chomsky, N. (2005). Three factors in language design. Linguistic inquiry, 36(1). https://doi.org/10.1162/0024389052993655

De Villiers, J. G., \& Pyers, J. E. (2002). Complements to cognition: a longitudinal study of the relationship between complex syntax and false-belief-understanding. Cognitive development, 17, 1037-1060. https://doi.org/10.1016/s0885-2014(02)00073-4

Ferreira, F. (1999). Syntax in language production: An approach using tree-adjoining grammars. L. Wheeldon (Ed.), aspects of language production (1999). Retrieved from: http://citeseerx.ist.psu.edu/viewdoc/download?doi=10.1.1.36.8465\&rep=rep1\&type=pdf

Hagoort, P. (2003). The interplay between syntax and semantics during sentence comprehension: ERP effects of combining syntactic and semantic violations. Journal of cognitive neuroscience, $6,15$. https://doi.org/10.1162/089892903322370807

Hanink, E. (2018). Postsyntactic inflection of the degree phrase in German. Proceeding of the Linguistic Society of America, 3, 23-1-15. https://doi.org/10.3765/plsa.v3i1.4308

Jackendoff, R. (1975). Morphological and semantic regularities in the lexicon. Language, 51, No.3. https://doi.org/10.2307/412891

Kratzer, A., \& Heim L. (1999). Semantics in generative grammar. Natural language semantics. Blackwell. https://doi.org/10.2307/417746

Munnich, E., Landau, B., Dosher, B. A. (2001). Spatial language and spatial representation: a cross-linguistic comparison. Cognition, 81, 171-207. https://doi.org/10.1016/s0010-0277(01)00127-5 
Singh, R. (2018). Norwegian and Persian spatial prepositions: a novel comparative and syntactic approach to the spatial prepositions. KAAV international journal of English, literature, and linguistics, 5, 1-8. Retrieved from: http://www.kaavpublications.org/journals/journal-5/article/article-1788.pdf

Slade, R., \& Ruffman, T. (2011). How language does (does not) relate to the theory of mind: a longitudinal study of syntax, semantics, working memory, and false belief. British journal of developmental psychology. https://doi.org/10.1348/026151004x21332

Slobin, D. I. (1966). From "thought and language" to "thinking for speaking". Rethinking language relativity. Cambridge: Cambridge university press. Retrieved from: https://philarchive.org/archive/SLOFTA 